\title{
Biotin-encoded Pullulan-Retinoic Acid Engineered Nanomicelles: Preparation, Optimization and In Vitro Cytotoxicity Assessment in MCF-7 Cells
}

\author{
F. HASSANZADEH, J. VARSHOSAZ1', G. A. KHODARAHMI², MAHBOUBEH ROSTAMI* AND FATEMEH HASSANZADEH \\ Isfahan Pharmaceutical Sciences Research Center and Department of Medicinal Chemistry, ${ }^{1}$ Department of \\ Pharmaceutics, ${ }^{2}$ Department of Medicinal Chemistry, School of Pharmacy and Pharmaceutical Sciences, Isfahan \\ University of Medical Sciences, Isfahan, Iran
}

\section{Hassanzadeh, et al.: Biotin-encoded Retinoic Acid Pullulan Nanomicelles}

\begin{abstract}
Biotin and retinoic acid grafted pullulan conjugate was synthesized using carbodiimide activation ester bond formation strategy for biotin targeted delivery of doxorubicin in breast cancer chemotherapy using micelle formulation. The conjugate structure was evaluated and confirmed by proton nuclear magnetic resonance and Fourier transform infrared spectroscopy. Critical micelle concentration of final conjugate was determined using the pyrene fluorescence method. Doxorubicin-loaded micelles were prepared using the direct dissolution method. Physical parameters of optimized micelles comprising particle size, zeta potential, drug loading efficacy, and drug release profile were determined. The results reveal that the nanomicelles have formed with mean particle size of $191 \mathrm{~nm}$ and zeta potential of $\mathbf{- 9 . 4 5} \mathbf{m v}$. The drug loading efficacy of $\mathbf{9 2 \%}$ and release efficiency of $81 \%$ during $24 \mathrm{~h}$, are also obtained. The structure of the micelles was studied by transition electron microscopy technique. The cytotoxicity of doxorubicin-loaded micelles was studied in MCF-7 cells using 3-(4,5-dimethylthiazol-2-yl)-2,5-diphenyltetrazolium bromide assay. The results of in vitro cell culture cytotoxicity assay showed that the doxorubicin-loaded biotin grafted retinoic acid-pullulan micelles were more cytotoxic in comparison to the non-targeted pullulan and free doxorubicin.
\end{abstract}

Key words: Targeted polymeric micelles; biotin-encoded; breast cancer; doxorubicin; pullulan

Being one of the most common causes of death, cancer keeps on taking millions of lives every year ${ }^{[1]}$. Various methods of therapy have been developed for treating cancer and even though there are many anticancer drugs available in the market, researches for a method or therapy with better and fewer side effects continue. The main problem with current drug use is the general cytotoxicity due to the distribution of these drugs in non-cancerous organs and cells. One of the best solutions to avoid this problem is the application of polymeric micelles (PMs). In this regard, during the past decade, various PM shave been widely evaluated as anticancer drug delivery systems to decrease drug degradation and loss, to decrease side effects and multiple drug resistance (MDR), and to increase drug bioavailability and drug accumulation in the required site of the body. PMs with unique properties such as high stability, low critical micelle concentration (CMC), biodegradability, small particle size and high loading capacity are suitable candidates to produce drug delivery systems ${ }^{[2,3]}$.

*Address for correspondence

E-mail:m.rostami@pharm.mui.ac.ir

September-October 2016
Pullulan as a nonionic polysaccharide is produced by fermentation of black yeast like Aureobasidium pullulans. Due to its special properties like nontoxicity, nonimmunogenicity, noncarcinogenicity, biodegradability and high water solubility, pullulan carriers are being developed for various biomedical applications including drug delivery ${ }^{[4,5]}$.

Producing micelles from pullulan requires conjugation with hydrophobic molecules to gain amphiphilic nature. Retinoic acid (RA) is an active metabolite of retinol (vitamin A) in the biological metabolic pathway and used in chemotherapy due to its role in the control of cell differentiation. Furthermore, its long carbon chain and the carboxylate group make it compatible for

This is an open access article distributed under terms of the Creative Commons Attribution-NonCommercial-ShareAlike 3.0 License, which allows others to remix, tweak, and build upon the work non-commercially, as long as the author is credited and the new creations are licensed under the identical terms.

Accepted 05 September 2016

Revised 29 August 2016

Received 29 May 2016

Indian J Pharm Sci 2016;78(5):557-565 
conjugating with pullulan to form PMs. Hence, in this study, RA was selected due to its anticancer activities against various cancers and its ability to control proliferation, differentiation and apoptosis of the cells along with its hydrophobic ability to form micelles with low $\mathrm{CMC}^{[6]}$.

The targeting effect of biotin has been studied on different cancer cell lines ${ }^{[7]}$, the overexpression of its receptors on the surface of the MCF-7 cell line has been proved $^{[8]}$. Recently, self-assembled folatebiotin-pullulan nanoparticles were prepared and their cytotoxicity was studied on KB cancerous cells by Wang et al.$^{[9]}$ Furthermore, Kim et al. ${ }^{[10]}$ synthesized biotin conjugated poly(ethylene oxide)/poly( $\varepsilon$ caprolactone) (PEG/PCL) nanoparticles and studied the paclitaxel loaded particles for their toxicity on normal human fibroblasts and HeLa cells ${ }^{[9-11]}$

To date, many anticancer drugs have been examined for treating breast cancer. Doxorubicin (DOX) is one of the most useful anthracycline antibiotics against breast cancer and some other cancers, but its use is limited due to its severe side effects such as myelosuppression and cardiotoxicity ${ }^{[12]}$.

In this study, biotin grafted RA-pullulan conjugate was prepared and applied to develop novel polymeric micelles for delivery of DOX to breast cancerous cells. DOX-loaded micelles were prepared by dialysis method. The physicochemical parameters of nanomicelles including particle size, zeta potential, surface morphology, loading and release efficiency, and in vitro cytotoxicity against MCF-7 cancerous cell line were examined. MCF-7 breast cancer cell line with overexpression of biotin receptors was chosen as the tumor model of breast cancer and the anticancer activity and the cellular uptake of DOX-loaded PMs were determined using MTT assay.

\section{MATERIAL AND METHODS}

The analytical grade pullulan (MW: $100 \mathrm{KD}$ ), 4-dimethylaminopyridine (DMAP), anhydrous dimethylsulfoxide (DMSO), 3-[4,5-dimethylthiazol2-yl]-2,5-diphenyl tetrazolium bromide (MTT) and ethyl alcohol were purchased from Merck Chemical Company, Germany and used as received. Dicyclohexylcarbodiimide (DCC), 1-ethyl-3-(3dimethylaminopropyl) carbodiimide (EDC), all-trans retinoic acid, pyrene, and dialysis tubing cutoff 2 and $10 \mathrm{kDa}$ as well as other compounds were purchased from Sigma Chemical Co., USA. DOX hydrochloride was provided from Hangzhou ICH Biopharm Co., Ltd., Zhejiang, China. Dulbecco's modified Eagle's medium and fetal bovine serum (FBS) were supplied from Gibco Laboratories, USA. The MCF-7 cell line was supplied by the Pasteur Institute, Iran.

\section{Synthesis of pullulan/RA conjugates (PR):}

As shown in the fig. $1,500 \mathrm{mg}(1.66 \mathrm{mmol})$ of RA was activated with the use of $640 \mathrm{mg}(3.33 \mathrm{mmol})$ of the EDC in anhydrous DMSO under the $\mathrm{N}_{2}$ atmosphere for $24 \mathrm{~h}$. Activated RA was then added drop wise to a solution of $300 \mathrm{mg}$ of pullulan in water $(10 \mathrm{ml})$ containing a few drops of DMSO and stirring continued for $48 \mathrm{~h}$. The resulted conjugated polymer was obtained by lyophilization and further purified using dialysis (membrane cut off $2 \mathrm{KD}$ ) against ethanol and deionized water.

\section{Synthesis of biotin grafted Pu/RA(PRB) micelles:}

To graft the biotin to $\mathrm{Pu} / \mathrm{RA}$ conjugate, $250 \mathrm{mg}(0.98$ mmol) biotin was activated with the use of $315 \mathrm{mg}$ (1.53 mmol) DCC and $125 \mathrm{mg}$ (1.02 mmol) DMAP in anhydrous DMSO (5 ml) under the $\mathrm{N}_{2}$ atmosphere. The solution was left to stir for $24 \mathrm{~h}$. After the removal of dicyclohexylurea (DCU) byproduct using centrifuge, the activated biotin was then added dropwise to a solution of $300 \mathrm{mg}$ of $\mathrm{Pu} / \mathrm{RA}$ in deionized water and stirring continued for a further $24 \mathrm{~h}$, the reaction path was provided in the fig. 1 . The resulted biotin targeted polymer was lyophilized and further purified using dialysis (membrane cut off $2 \mathrm{KD}$ ) against ethanol, phosphate buffer $\mathrm{pH} 7.8$, acetate buffer $\mathrm{pH} 6.2$ and deionized water.

The structure of final macromolecule was confirmed by FTIR and ${ }^{1}$ HNMR spectroscopy. The ${ }^{1} \mathrm{HNMR}$ spectrum of the conjugate was recorded on $(400 \mathrm{MHz}$, AC-80, Bruker Biospin, Germany) using DMSO: D2O (1:9) as solvent (fig. 2). The FTIR spectra were recorded on a Fourier transform infrared spectrometer (Rayleigh, WQF-510/520, and China) as KBr pellets in the range of $4000-400 \mathrm{~cm}^{-1}$. Finally, the degree of substitution (DS) was calculated using the ${ }^{1} \mathrm{HNMR}$ spectrum of final conjugate. In this regards, the peaks positioned at 2.81 and $5.71 \mathrm{ppm}$ for the Biotin and the RA, respectively, and the reference peak of pullulan at $5.32 \mathrm{ppm}$ in Biotin-grafted $\mathrm{Pu} / \mathrm{RA}$ conjugate were used to determine the degree of substitution (DS) of Biotin and RA on pullulan scaffold.

\section{Preparation of DOX-loaded PRB micelles:}

To prepare the DOX-loaded Biotin-grafted Pu/RA 


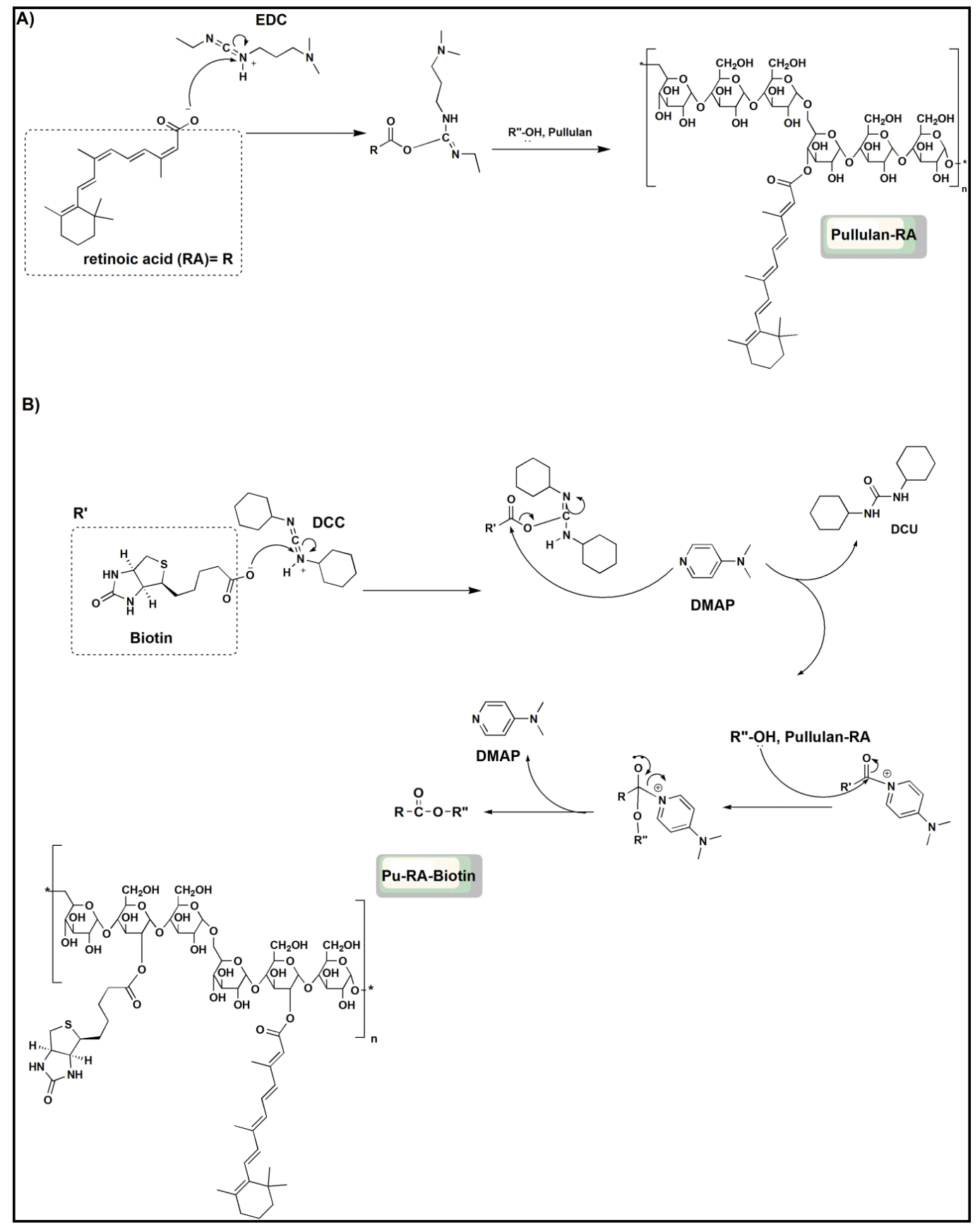

Fig. 1: Reaction for synthesis of biotin encoded retinoic acid-pullulan nanomicelles.

A. chemical syntheses of pullulan/RA conjugate; B. chemical synthesis of biotin grafted Pu/RA.

micelles by direct dissolution method, $1.5 \mathrm{mg}$ of each conjugate was dissolved in $15 \mathrm{ml}$ of deionized water and sonicated for $20 \mathrm{~min}$. A sample of $5 \mathrm{ml}$ was separated for further use as blank; $1 \mathrm{mg}$ of DOX was added to the rest of the solution. This solution was stirred at $4000 \mathrm{rpm}$ in $25^{\circ}$ for an hour and then $2 \mathrm{~min}$ sonication was done by probe sonication (Bandelin, HD 3200 Germany) using TT13 probe at 30\% power amplitude. All experiments were done in triplicate.

\section{CMC of PRB micelles:}

To determine the CMC value of the PRB micelles, pyrene is used as a hydrophobic fluorescent probe in fluorescence spectroscopy, which preferably can be disposed into the hydrophobic core of the micelles. When the pyrene transits from a hydrophilic medium (water) to a hydrophobic environment (micelles core), the excitation spectrum shifts to longer wavelengths. The CMC value can be determined by evaluation of the changes in the ratio of the pyrene excitation intensity at $\lambda_{\mathrm{ex}}=662 \mathrm{~nm}$ (I662) and $\lambda_{\mathrm{ex}}=386 \mathrm{~nm}$ (I386, for pyrene within the hydrophobic micelles core) using recorded excitation spectra at $\lambda_{\mathrm{em}}=329 \mathrm{~nm}$. Therefore, 
the excitation spectra of pyrene in water and in the presence of the biotin-grafted $\mathrm{Pu} / \mathrm{RA}$ micelles with a concentration higher than the CMC were recorded. Briefly, the micellar aqueous solutions of PRB with increasing concentrations from 2.5 to $100 \mu \mathrm{g} / \mathrm{ml}$ were prepared. One millilitre of a pyrene solution in acetone $\left(6.0 \times 10^{-6} \mathrm{M}\right)$ was mixed with $10 \mathrm{ml}$ of each polymer solution to reach the final pyrene concentration of $6.0 \times 10^{-7} \mathrm{M}$ in each sample solution. The samples were shaken at $37^{\circ}$ before the analysis for $24 \mathrm{~h}$. The fluorescence intensities were measured at $\lambda_{\mathrm{em}}=329$ $\mathrm{nm}$ and $\lambda_{\text {ex }}=662$ and $386 \mathrm{~nm}$ by spectrofluorimeter (LS-3, PerkinElmer, USA). The change in the ratio of fluorescence intensity (I662/I386) of pyrene to the logarithmic scale of biotin-grafted $\mathrm{Pu} / \mathrm{RA}$ conjugate concentration was plotted for determining the $\mathrm{CMC}^{[13]}$.

\section{Particle size and zeta potential measurements:}

The mean particle size and zeta potential of PRB micelles were measured by photon correlation spectroscopy (PCS) by a Zetasizer (Zetasizer-ZEN 3600 Malvern Instrument Ltd., Worchestershire, UK). All the particle sizes were measured in deionized water without dilution directly after micelles preparation using a He-Ne laser beam at $658 \mathrm{~nm}$ with a scattering angle of $90^{\circ[14]}$.

\section{Entrapment efficiency of DOX in the micelle:}

To determine the entrapment efficiency of DOX in the PRB micelles, briefly, a $300 \mu 1$ of PRB nanomicelles was centrifuged using normal centrifugal tubes for 5 min at $10000 \mathrm{rpm}$ to separate the aqueous solution. The concentration of free DOX in the supernatant was determined using the UV/visible spectrophotometer (UV-mini-1240 CE-Shimadzu, Japan) at $\lambda_{\max } 249 \mathrm{~nm}$ in distilled water. A solution including all components except for the DOX was used as the blank. The drug entrapment efficiency was calculated using the difference between the first total and the final free DOX concentration. The entrapment efficiency of DOX in PRB nanomicelles was calculated using following Eqn. ${ }^{[15]}, \mathrm{EE}(\%)=($ DOX total-DOX supernatant/DOX total)x 100 .

\section{In vitro drug release study:}

To evaluate the in vitro release of DOX from the prepared PRB micelles, the phosphate buffer solution (PBS) pH 7.4 with the concentration of $0.05 \mathrm{M}$ was prepared to use as the in vitro release medium in the DOX release study. Two millilitre of DOX- loaded micellar dispersion was placed in the dialysis membrane bag (cutoff $2 \mathrm{KDa}$ ) and the bag was sunk fully in $15 \mathrm{ml}$ of release medium under stirring at $37^{\circ}$. At specified time intervals, the samples were taken and the concentration of DOX released in the medium was determined by UV/Vis spectrophotometer at $\lambda_{\text {max }}=501$ $\mathrm{nm}$ until the plateau phase was reached.

\section{Transition electron microscopy (TEM):}

To observe the surface morphology and the shapes of nanomicelles in dried state, a drop of freshly prepared well-dispersed micelles was placed on a 300 mesh carbon-coated copper grid and the grid was left to dry at room temperature. Micrographs were taken with different levels of magnification with an $80 \mathrm{kV}$ accelerating voltage applying a Transmission Electron Microscope (Zeiss, EM10C, Germany).

\section{Cell viability assay:}

MCF-7 cell lines which overexpress biotin receptor ${ }^{[8]}$ were used in this study. The cells were cultured in RPMI1640 medium containing 10\% FBS and 1\% antibiotics mixture of penicillin $(5000 \mathrm{U} / \mathrm{ml})$ and streptomycin $(5000 \mu \mathrm{g} / \mathrm{ml})$ at $37^{\circ}$ under $5 \% \mathrm{CO}_{2}$ content with high humidity. First cellular suspensions of MCF7 cells at a density of $5 \times 10^{4}$ cells $/ \mathrm{ml}$ were prepared. Then, $180 \mu \mathrm{l}$ of the suspension was seeded into each well of 96-well culture plate (SPL Lifescience, Korea) for each cell line and incubation was done (6500, Napco, France) for $24 \mathrm{~h}$ at $37^{\circ}$ in $5 \% \mathrm{CO}_{2}$ with proper humidity before cell viability tests. Then each raw was treated with $20 \mu \mathrm{l}$ of five concentrations $(0.00058$, $0.0058,0.058,0.58$ and $5.8 \mu \mathrm{g} / \mathrm{ml}$ ) of free DOX solutions (as positive control), $20 \mu \mathrm{l}$ of culture medium (as negative control), $20 \mu \mathrm{l}$ of drug-loaded micelles of PRB with or without biotin ligand containing the same five concentrations of DOX $(0.00058,0.0058$, $0.058,0.58$ and $5.8 \mu \mathrm{g} / \mathrm{ml}$ ) and $20 \mu \mathrm{l}$ of blank micelles of PRB with or without biotin ligand at the same five concentrations of the drug-loaded micelles dispersion. The plate was then incubated for further $48 \mathrm{~h}$ and after that $20 \mu$ of MTT solution ( $5 \mathrm{mg} / \mathrm{ml}$ of PBS) was added and incubated for $3 \mathrm{~h}$, the cell medium was centrifuged (MIKRO200, Hettich, Germany) at $1800 \mathrm{rpm}$ and removed cautiously so that the produced crystalline formazan not be lost. Then, $150 \mu$ l of DMSO was added to dissolve the formazan crystals and the concentration of formazan in each row was measured separately using an enzyme-linked immunosorbent assay (ELISA) plate reader (Awareness, USA) at $570 \mathrm{~nm}$. Cell viability for each sample was determined using following Eqn., \% 
cell survival $=(($ mean abs. of the test-mean abs. of the blank $) /($ mean abs. of negative control-mean abs. the blank))x100

\section{RESULTS AND DISCUSSION}

The path of the chemical reaction between RA, biotin and pullulan is shown in fig. 1. PRB micelle was successfully prepared through the esterification reaction between RA and biotin carboxylic group and pullulan hydroxyl groups. The structure of final conjugate was confirmed by FTIR and ${ }^{1}$ HNMR spectra (figs. 2 and 3). Fig. 2 shows the FTIR spectra of biotin grafted $\mathrm{Pu} / \mathrm{RA}$ (PRB) (fig. 2a), Pu/RA (fig. 2b), pullulan (fig. 2c), biotin (fig. 2d) and RA (fig. 2e). Fig. 3 shows the ${ }^{1} \mathrm{HNMR}$ spectra of biotin grafted $\mathrm{Pu} / \mathrm{RA}$ (PRB) (fig. 3a) in comparison with its components pullulan (fig. 3c), biotin (fig. 3d) and RA (fig. 3e). Furthermore, the ${ }^{1} \mathrm{HNMR}$ spectra of $\mathrm{Pu} / \mathrm{RA}$ conjugate $(\mathrm{PR})$ is provided in fig. $3 b$.

A fluorescence method was used to estimate the copolymer concentration at which the first micellization takes place. The emission spectrum of pyrene in water and in the presence of synthesized macromolecule at $329 \mathrm{~nm}$ showed an excitation wavelength for it at

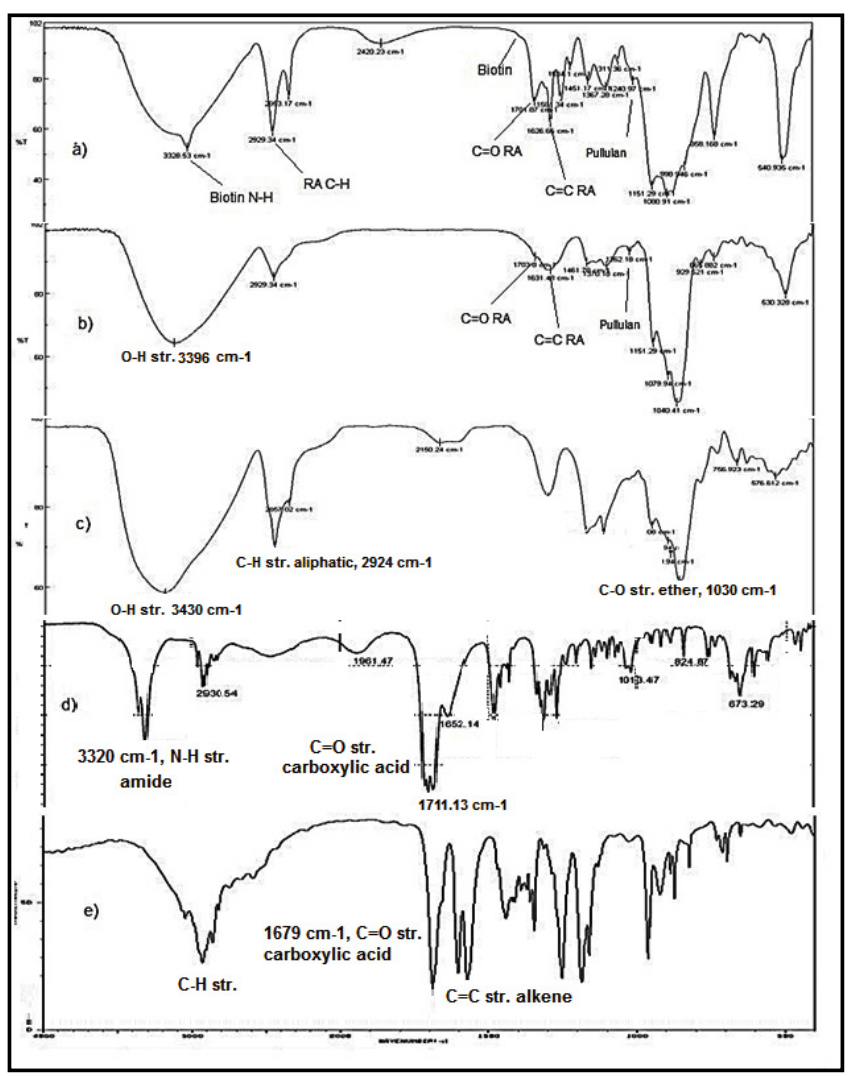

Fig. 2: FTIR analysis of biotin grafted pullulan/RA conjugate. a) pullulan-biotin-RA (PRB), b) pullulan/RA conjugate, c) pullulan, d) biotin, e) retinoic acid.

September-October 2016

Indian Journal of Pharmaceutical Sciences

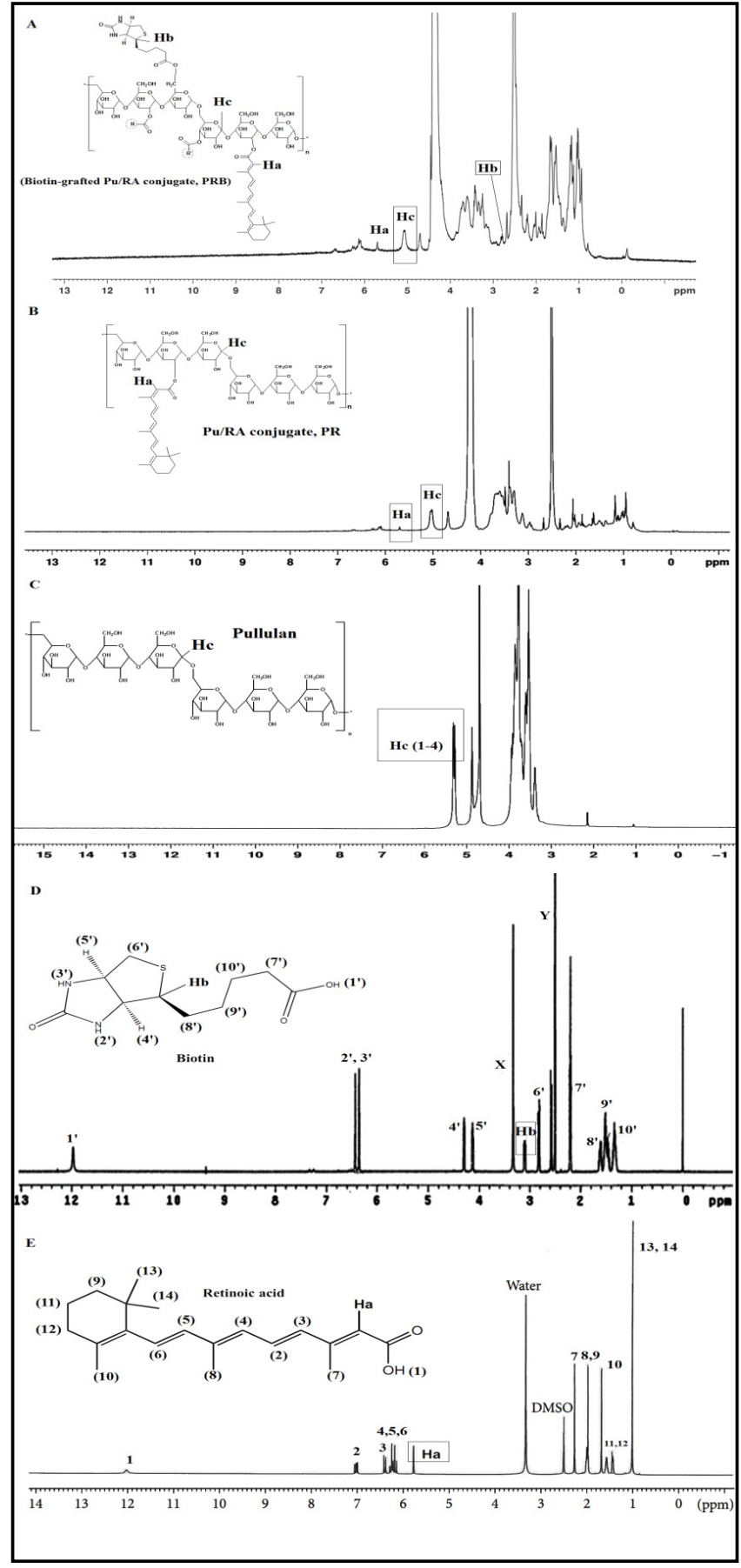

Fig. 3: ${ }^{1}$ HNMR analysis of biotin grafted pullulan-RA conjugate. a) pullulan-biotin-RA (PRB), b) pullulan/RA, c) biotin, d) pullulan, e) retinoic acid.

$662 \mathrm{~nm}$ and $386 \mathrm{~nm}$ in the presence of the synthesized macromolecule, the plot of the fluorescence intensity ratio (I662/I386) versus the logarithm of the concentration of the micellar aqueous solutions is presented in fig. 4.

The physicochemical parameters of DOX-loaded PRB micelles including size, zeta potential and loading efficiency are shown in Table 1. Fig. 5 shows the release 


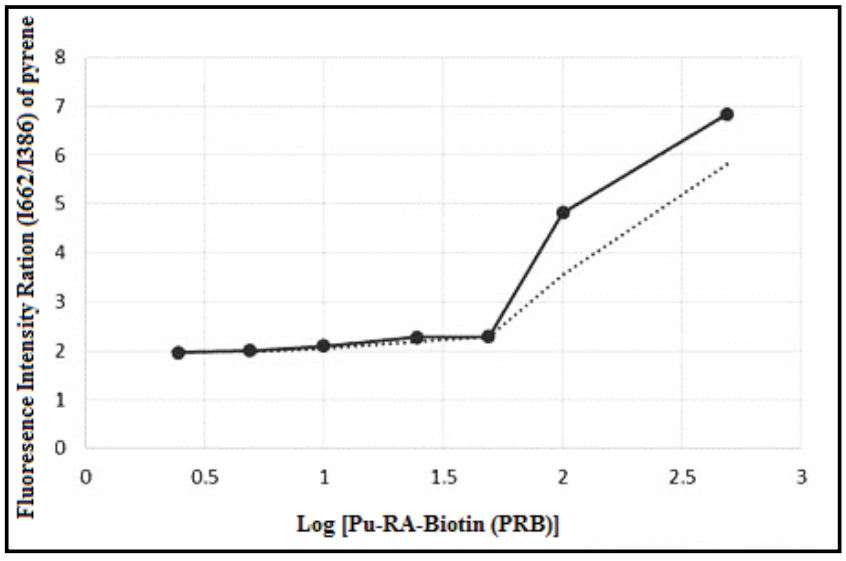

Fig. 4: Plot of the fluorescence intensity ratio (I662/I386) versus the $\log$ of the concentration of the micellar aqueous solutions. Determination of the micelles CMC of PRB micelle by plotting the ratios of fluorescence intensity of an emission wavelength of 662 to that of excitation wavelength of $329 \mathrm{~nm}$ in terms of concentration logarithm.

profiles of DOX versus time for PRB and PR micellar formulations. TEM micrographs of PRB nanoparticles with different levels of magnification are provided in fig. 6. MCF-7 Cell viability results in the presence of different test groups have been collected in figs. $7 \mathrm{a}$ and $7 \mathrm{~b}$.

As you can see in fig. 2a, the emergence of absorption bands around 3328 and around $292 \mathrm{~cm}^{-1}$ which respectively are attributed to stretching vibration of biotin amide $\mathrm{N}-\mathrm{H}$ bond and stretching vibration of alkene $\mathrm{C}-\mathrm{H}$ bonds of $\mathrm{RA}$ in biotin-grafted $\mathrm{Pu} / \mathrm{RA}$ in comparing to pullulan FTIR spectrum reveals the presence of the RA and the biotin (fig. 2e). Along with this evidence, presence of the strong absorption band around the 1700 and $1626 \mathrm{~cm}^{-1}$ (fig. 2a) for newly formed ester $\mathrm{C}=\mathrm{O}$ moieties are another proofs to the synthesis of the desired conjugate which confirms the presence of some newly attached groups to pullulan which is not seen in the fig. $2 \mathrm{c}$, furthermore, the presence of other C-O stretching characteristic absorption bands of pullulan around $1090 \mathrm{~cm}^{-1}$ in the final conjugate spectra is the other important evidence for the accurate synthesis. In the other words, the changes in the final conjugate spectral feature compared to its components spectral patterns convinced us to successful synthesis of desired conjugate, other supporting valuable evidences were extracted from the ${ }^{1} \mathrm{H}$ NMR spectra as discussed in continue.

The ${ }^{1} \mathrm{HNMR}$ of PRB (fig. 3a) in comparison to pullulan (fig. 3c) has new signals at around 1.00 (1 signal), 1.401.50 (multiple signals), 1.70 (1 signal), 2.00 (1 signal), 2.30 (1 signal), 5.70 (1 signal), 6.00-7.20 (4 signals) $\mathrm{ppm}^{[16]}$ and 1.10-1.80 (3 signals), 2.10-2.90 (3 signals), 3.10 (1 signal), 4.10-4.20 (2 signals), 6.20-6.40 (2
TABLE 1: PHYSICAL PROPOERTIES OF DOXORUBICIN FROM TARGETED AND NON-TARGETED MICELLES

\begin{tabular}{ccccc}
\hline Sample & $\begin{array}{c}\text { Particle } \\
\text { size }(\mathrm{nm})\end{array}$ & $\begin{array}{c}\text { Zeta potential } \\
(\mathrm{mv})\end{array}$ & EE (\%) & RE $_{24}(\%)$ \\
\hline PR & $235 \pm 8.7$ & -9.60 & $75.32 \pm 0.30$ & $87 \pm 4.3$ \\
PRB & $191 \pm 7.3$ & -9.45 & $92.26 \pm 0.78$ & $81 \pm 2.5$ \\
\hline
\end{tabular}

$(\mathrm{n}=3, \mathrm{SD} \pm$ Average $)$

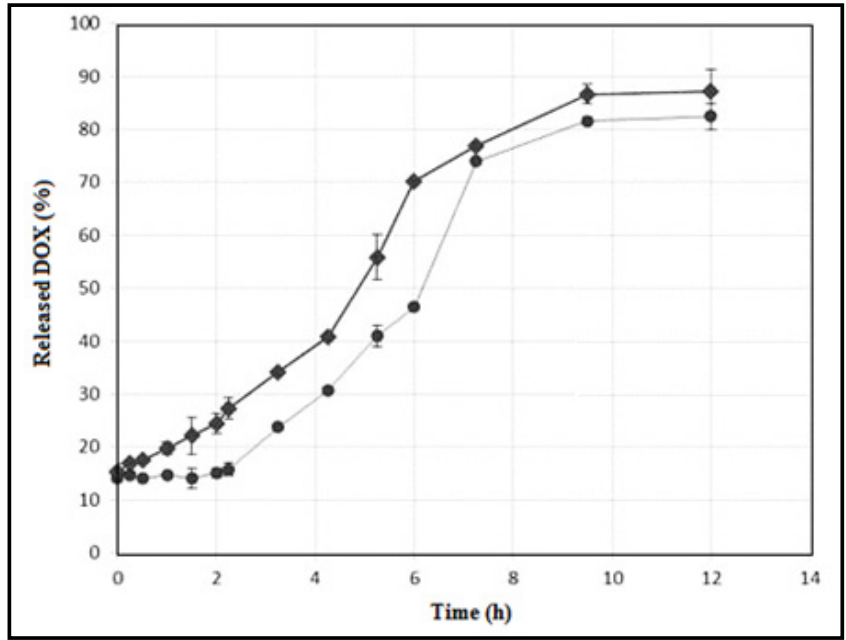

Fig. 5: Percentage release of doxorubicine from nanomicelles. Percentage release from (-๑-) PRB micelles and (-^) PR micelles.

signals) $\mathrm{ppm}^{[17]}$ which all attributed respectively to RA and biotin components along with disappearance of carboxylic signals of both RA and biotin around $12 \mathrm{ppm}$. For more explanation, we can point out that, all expected spectral components which ensure us about the accurate synthesis of PRB conjugate were obtained by analysis the ${ }^{1} \mathrm{HNMR}$ spectra of PRB. All mentioned signals along with the presence of pullulan characteristic signals around 3.00-4.00 ppm and 4.90 (H1, 1-6 linkage) and 5.30 (H1, 1-4 linkage) ppm as assigned in fig. $2 \mathrm{c}^{[18,19]}$ were the best convincing evidences for the successful synthesis of PRB. With another look, the covalent bonding of RA and biotin to pullulan was confirmed by the presence of related peaks of RA and biotin in PRB spectra after $72 \mathrm{~h}$ dialysis in different mediums along with the slight changes in the displacement of reference peaks for RA $(5.70 \mathrm{ppm})$ and biotin $(2.80 \mathrm{ppm})$ in the final conjugate which respectively appeared in $5.80 \mathrm{ppm}$ and 3.10 ppm in the corresponding spectra of RA (fig. 3e) and biotin (fig. 3d). In ${ }^{1} \mathrm{HNMR}$ spectra of RA a singlet peak attributed to Ha of RA adjuvant to the carboxylic group, is observed at $5.80 \mathrm{ppm} \mathrm{Ha}$, which is displaced downfield at $5.70 \mathrm{ppm}$ due to the esterification. In the same way, a singlet peak attributed to $\mathrm{Hb}$ of biotin which in ${ }^{1} \mathrm{HNMR}$ of biotin is appeared at $3.10 \mathrm{ppm}$, now in the 


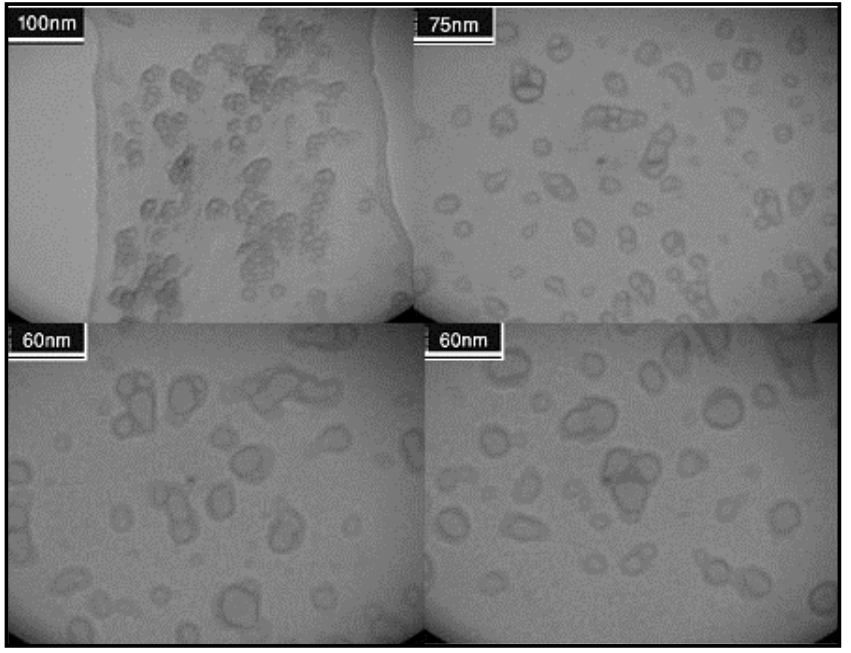

Fig. 6: TEM images of doxorubicine-loaded PRB micelles.

final conjugate is moved up-field around the $2.80 \mathrm{ppm}$.

To calculate the DS for each of the RA and biotin components, the singlet peak at $5.70 \mathrm{ppm}$ (Ha, equivalent to 1 proton as shown in the fig. $3 \mathrm{e}$ ) and the peak at $2.80 \mathrm{ppm}(\mathrm{Hb}$, equivalent to 1 proton as shown in the fig. 3d), respectively attributed to the RA and biotin were chosen as the reference peak in comparison to the pullulan the reference peak around $5.10 \mathrm{ppm}(\mathrm{Hc}$, equivalent to $66 \mathrm{H}$ per 100 glucose units, fig. 3c) and the DS for each of them was calculated using following Eqns.,

$\operatorname{DS}(\mathrm{RA})(\%)=\frac{\frac{\text { intergral of peak at } 5.70 \mathrm{ppm} \text { for RA }(\mathrm{Ha})}{1}}{\frac{\text { intergral of peak at } 5.10 \mathrm{ppm} \text { for pullulan }(\mathrm{Hc})}{66}}=17.2 \%$

$\operatorname{DS}\left(\right.$ Biotin) $(\%)=\frac{\frac{\text { intergral of peak at } 2.80 \mathrm{ppm} \text { for biotin }(\mathrm{Hb})}{1}}{\frac{\text { intergral of peak at } 5.10 \mathrm{ppm} \text { for pullulan }(\mathrm{Hc})}{66}}=13.6 \%$

Plot of the ratio of fluorescence intensity in two wavelengths (I662/I386) versus the logarithm of the concentration of the micellar aqueous solutions showed a sigmoidal curve in which at concentrations lower than the $\mathrm{CMC}$, there is no significant change in fluorescence intensity, while in the concentrations higher than the $\mathrm{CMC}$, a linear increase in the ratios was observed with increase in PRB concentration. The $\mathrm{CMC}$ value was obtained from the intersection of the line with an almost gentle slope in the changes of the ratio of I662/I386 and the line with a steady increase in the ratio value (fig. 4). The estimated CMC value is 60 $\mu \mathrm{g} / \mathrm{ml}$; in the aqueous environment. The hydrophobic RA moieties of PRB were assembled themselves in hydrophobic cores, while these cores were surrounded by the hydrophilic pullulan scaffold. Micelle solutions are diluted in the body and they are expected to be disassociated. The more stable micelles in the body fluids are those with the lower CMC value ${ }^{[20]}$. In this research, PRB conjugate had the CMC value $(60 \mu \mathrm{g} /$ $\mathrm{ml}$ ), around 40 times lower than the concentrations needed to form micelles with low-molecular weight surfactants, e.g., sodium dodecyl sulfate (SDS) which has a $\mathrm{CMC}$ of $2.3 \mathrm{mg} / \mathrm{ml}^{[21]}$. This result indicates that the PRB conjugate can form quite stable micelles even in highly diluted solutions.

The size and size distribution of PRB micelles were studied by dynamic light scattering (DLS). As table 1 displays, both targeted and untargeted polymers can form drug-loaded micelles in acceptable sizes, also biotin targeting may decrease the particle size and zeta potential of the micelles ${ }^{[22]}$.

The hydrophobic forces that dispatch the hydrophobic chain into the core and the volume repulsion between the chains principally establish the micellar size ${ }^{[23]}$. The zeta potential of PRB and PR are both negative in the range of $-9 \mathrm{mV}$, this negative zeta potential must have originated from the presence of numerous unreacted hydroxyl groups on the final conjugates, as expected and in the same studies was reported ${ }^{[24]}$, this residual charge of the systems can convinced us to formation of stable micelles.

The hydrophobic core of the micelles acts as a vehicle to increase the solubility and stability of hydrophobic drugs. The capacity of the hydrophobic core is limited. The drugs are loaded by the formation of non-covalent bonds into the core. The amount and efficiency of drug loading depend on factors comprising the nature of the drug, the nature of the hydrophobic core, the size of the micelles core and the drug concentration, in the high ratio of the drug to polymeric micelles, usually the loading is reduced ${ }^{[25]}$. In this study, DOX was readily loaded in PRB micelles in the ratio of $100 \%$ to PRB and entrapment efficiency was about $92 \%$.

The drug release from micelles depends on the nature of the drug, the drug concentration, distribution coefficient of a drug between the hydrophobic core and the release medium and the polymer degradation rate ${ }^{[26]}$. In this study, $81 \%$ of loaded DOX were released from the micelles within the first $12 \mathrm{~h}$. As can be seen from fig. 6 , in the same conditions, biotin targeted micelle releases its DOX content in a better controlled rate than the non-targeted ones.

As can be seen in fig. 6, TEM image shows a separate and distinct distribution of spherical-shaped micelles well and also the particle size is slightly smaller than 
the results obtained by DLS (Malvern ZetaSizer), this difference is due to the fact that the TEM imaging was performed out of the solution in which hydration and other types of interactions can take place, in other words, in the DLS analysis condition the hydrodynamic diameter is measured which is greater than the estimated size by $\mathrm{TEM}^{[27]}$.

Fig. 7a shows the results of MCF-7 cell viability by MTT assay in the presence of free DOX, DOX-loaded targeted micelles and DOX-loaded non-targeted micelles. It can be readily determined that the $\mathrm{IC}_{50}$ for free DOX is about $0.163 \mu \mathrm{M}$, whereas for PR and PRB is about 0.080 and $0.050 \mu \mathrm{M}$, respectively. To determine the significance of difference, all data obtained from cell culture were analyzed by SPSS 22 software.

From these data we can conclude that, in all concentrations, there is a significant difference between cytotoxicity of the drug-loaded micelles, bare micelles and negative control $(\mathrm{P}<0.05)$. At concentrations lower than the $\mathrm{IC}_{50}$ of free DOX, percentage of survival in wells of the DOX-loaded targeted micelles is less than that of the free DOX and there is a significant difference between the two groups. According to the results it can be concluded that the biotin targeting has been successful and by this approach, the required DOX dose to control MCF-7 cancerous cells gets decreased.

In our research, the $\mathrm{IC}_{50}$ of DOX-loaded targeted (PRB) and non-targeted (PR) micelles were respectively 0.050 and $0.080 \mu \mathrm{M}$, which both of them are lower than the $\mathrm{IC}_{50}$ of free DOX. At the concentrations above the $\mathrm{IC}_{50}$, there is no noteworthy difference between the free DOX and DOX loaded in micelles ( $P>0.05)$, this may be due to the saturation in binding of targeted micelles to cancerous cells more than the normal cells. These results showed that DOX loaded in biotin-targeted micelles was more cytotoxic than free DOX and DOXloaded non-targeted micelles (fig. 7b). In other words, in all concentrations below the $\mathrm{IC}_{50}$, targeted micelle could be applied to reduce the side effects by binding to cancer cells rather than the normal cells.

In conclusion, PRB conjugate was successfully synthesized with the aid of carbodiimide strategy. The nanomicelles were prepared for targeted delivery of DOX to MCF-7 breast cancer cell line with a narrow distribution in size. The good negative zeta potential give the micelles disperse ability and good colloidal stability in water. Entrapment efficiency of DOX was high and the release rate in the first hours was fast and
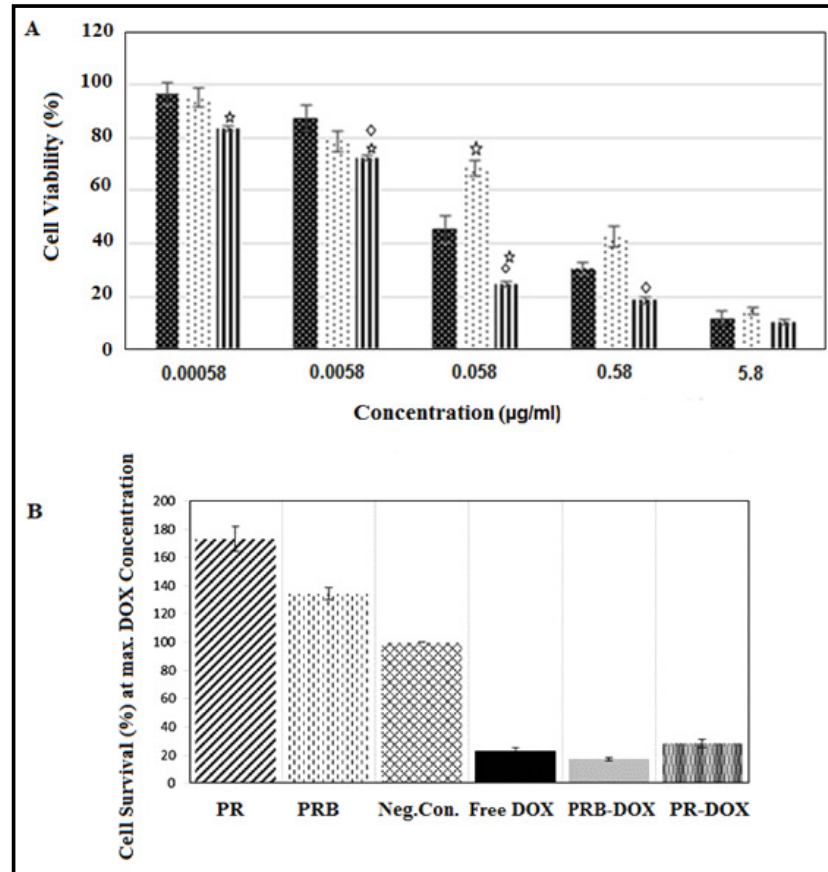

Fig. 7: MCF-7 cell viability results in the presence of different test groups.

A. results of comparing the viability of cells in percent per loading, with the positive control (DOX), (star sign: significant difference between free doxorubicin (positive control) and drug loaded micelles $(\mathrm{P}<0.05)$, diamond sign: significant difference between targeted and untargeted micelles $(P<0.05))$ DOX IIII PRB micelles, PR Micellec (non-targeted). B. Percentage of cell survival with different test groups $\left(>\mathrm{IC}_{50}\right.$ of DOX, $5.8 \mu \mathrm{g} / \mathrm{ml})$.

quantitatively. The DOX-loaded PRB micelles were significantly more cytotoxic than the free DOX and DOX-loaded $\mathrm{Pu} / \mathrm{RA}(\mathrm{PR})$ and this may be due to the active endocytosis of the DOX via the biotin receptors over expressed on the surface of MCF-7 cancerous cell lines. These results should be checked in vivo to confirm the promising results on the cell culture. However, in our opinion, the results of this study are promising results in the treatment of cancer.

\section{Financial support and sponsorship:}

Nil.

\section{Conflicts of interest:}

The author declares no competing interests.

\section{REFERENCES}

1. DeSantis C, Ma J, Bryan L, Ahmedin J. Breast cancer statistics. CA Cancer J Clin 2013;64:52-62.

2. Uhrich KE, Cannizzaro SM, Langer RS, Shakesheff KM. Polymeric systems for controlled drug release. Chem Rev 1999;99:3181-198.

3. Kedar U, Phutane P, Shidhaye S, Kadam V. Advances in 
polymeric micelles for drug delivery and tumor targeting. Nanomedicine 2010;6:714-29.

4. Singh RS, Saini GK, Kennedy JF. Pullulan: Microbial sources, production and applications. Carbohyd Polym 2008;73:515-20.

5. Kumar D, Saini N, Pandit V, Ali S. An insight to pullulan: a biopolymer in pharmaceutical approach. Int J Basic Appl Sci 2012;1:202-19.

6. Lee J, Jeong D, Seo S. Biodegradable nanogel based on all Trans retinoic acid/pullulan conjugate for anticancer drug delivery. J Pharm Invest 2013;42:4363-69.

7. Shuyi C, Xianrui Z, Jingyi C, Jin C, Larisa K, Stanislaus SW, et al. Mechanism-based tumor-targeting drug delivery system validation of efficient vitamin receptor-mediated endocytosis and drug release. Bioconjugate Chem 2010;21:979-87.

8. So YK, Seung HC, Young ML. Biotin-conjugated block copolymeric nanomicelles as tumor-targeted drug delivery systems. Macromol Res 2007;15:646-55.

9. Wang M, Huang M, Wang J, Ye M, Deng Y, Li H, et al. Facile one-pot synthesis of self-assembled folate-biotin-pullulan nanoparticles for targeted intracellular anticancer drug delivery. J Nanomat 2016;2016:5752921.

10. Kim SY, Cho SH, Lee YM. Biotin-conjugated block copolymeric nanoparticles as tumor-targeted drug delivery systems. Macromol Res 2007;15:646-55.

11. Li H, Sun Y, Liang J, Fan Y, Zhang X. pH-Sensitive pullulanDOX conjugate nanoparticles for co-loading PDTC to suppress growth and chemoresistance of hepatocellular carcinoma. J Mater Chem B 2015;3:8070-78.

12. Keizer HG, Pinedo HMG, Schuurhuis J, Joenje H. Doxorubicin (adriamycin): a critical review of free radical-dependent mechanisms of cytotoxicity. Pharmacol Ther 1990;47:219-31.

13. Wolszczak M, Miller J. Characterization of non-ionic surfactant aggregates by fluorometric techniques. J Photochem Photobiol A: Chem 2002;147:45-54.

14. Riess G. Micellization of block copolymers. Prog Polym Sci 2003;28:1107-70.

15. Varshosaz J, Hassanzadeh F, Sadeghi H, Andalib S. Synthesis of octadecylamine-retinoic acid conjugate for enhanced cytotoxic effects of 5-FU using LDL targeted nanostructured lipid carriers. Eur J Med Chem. 2012;54:429-38.

16. Varshosaz J, Hassanzadeh F, Aliabadi HS, Banitalebi M,
Rostami M, Nayebsadrian M. Novel worm-like amphiphilic micelles of folate-targeted cyclodextrin/retinoic acid for delivery of doxorubicin in KG-1 cells. Colloid Polym Sci 2014;292:2647-62.

17. Mitsuhiko I, Kunio H. Application of two dimensional NMR spectroscopy to the complete analysis of the $1 \mathrm{H}$ and $13 \mathrm{C}$ NMR spectra of d-Biotin in aqueous solution. Magn Reson Chem 1982;20:266-73.

18. Sunamoto J, Akiyoshi K, Hosotani R, Hayashi A, Fukui H. High purity polysaccharide containing a hydrophobic group and process for producing it. US Patent 2003;65665516 B1.

19. Sasaki Y, Tsuchido Y, Sawadaa S, Akiyoshi K. Construction of protein-crosslinked nanogels with vitamin B6 bearing polysaccharide. Polym Chem 2011;2:1267-70.

20. Bian F, Jia L, Yu W, Liu M. Self-assembled micelles of n-phthaloylchitosan-g-polyvinylpyrrolidone for drug delivery. Carbohyd Polym 2009;76:454-59.

21. Shirahama K, Kashiwabara T. The CMC-decreasing effects of some added alcohols on the aqueous sodium dodecyl sulfate solutions. J Colloid Interface Sci 1971;36:65-70.

22. Park KH, Kang DM, Na K. Physicochemical characterization and carcinoma cell interation of self-organized nanogels prepared from Polysaccharide/Biotin conjugates for development of anticancer drug carrier. J Microbiol Biotechnol 2006;16:1369-76.

23. Ferreira SA, Coutinho PJG, Gama FM. Synthesis and characterization of self-assembled nanogels made by pullulan. Materials 2011;4:601-20.

24. Sarika PR, James NR, Nishna N, Anil Kumar PR, Raj DK. Galactosylated pullulan-curcumin conjugate micelles for site specific anticancer activity to hepatocarcinoma cells. Colloids Surf B Biointerfaces 2015;133:347-55.

25. Mahmoud AA, El-Feky GS, Kamel R, Awad GEA. Chitosan/ sulfobutylether- $\beta$-cyclodextrin nanoparticles as a potential approach for ocular drug delivery. Int J Pharm 2011;413:229-36.

26. Singh A, Worku ZA, Mooter GV. Oral formulation strategies to improve solubility of poorly water-soluble drugs. Expert Opin Drug Deliv 2011;8:1361-78.

27. Zhang HZ, Li XM, Gao FP, Liu LR, Zhou ZM, Zahng QQ. Preparation of folate-modified pullulan acetate nanoparticles for tumor-targeted drug delivery. Drug Deliv 2010;17:48-57. 